\title{
PRELIMINARY RESULTS OF LARGE EDDY SIMULATIONS OF A HYDROCYCLONE
}

\author{
F. J. Souza ${ }^{a}$, ABSTRACT \\ and A. Silveira Neto ${ }^{b}$ \\ Subgrid-scale modeling, which characterizes Large Eddy Simulation (LES), has been used \\ to predict the behavior of a water-fed hydrocyclone operating without an air core. The \\ governing equations were solved by a fractional step method on a staggered grid. The \\ ${ }^{a}$ Universidade Federal de Uberlândia \\ Faculdade de Engenharia Mecânica \\ Av. João Naves de Ávila, 2160 \\ Uberlândia, Minas Gerais, Brasi \\ fjsouza@mecanica.ufu.br \\ ${ }^{b}$ Universidade Federal de Uberlândia \\ Faculdade de Engenharia Mecânica \\ Av. João Naves de Ávila, 2160 \\ Uberlândia, Minas Gerais, Brasil \\ Smagorinsky subgrid-scale model was employed to account for turbulent effects. Numerical \\ results actually capture the main features of the flow pattern and agree reasonably well \\ with experiments, suggesting that LES represents an interesting alternative to classical \\ turbulence models when applied to the numerical solution of fluid flows within \\ hydrocyclones \\ Keywords: hydrocyclones, Large Eddy Simulation, numerical solution.
}

\section{INTRODUCTION}

Hydrocyclones (Svarovsky, 1984) constitute an important class of devices intended for the separation of solid-liquid suspensions. The principle employed is centrifugal sedimentation, i.e., the particles in the suspension are submitted to a centrifugal field, which provokes their separation from the fluid. Unlike centrifuges, which make use of the same principle, hydrocyclones do not present moving parts, require low installation and maintenance investment and are simple to operate. Hence, these devices are widely utilized in mineral, chemical, petrochemical, textile and metallurgical industries.

Despite the simplicity of its construction, the hydrocyclone displays a quite complex internal hydrodynamic behavior, including features as high preservation of vorticity, vortex breakdown and reverse flow (Dyakowsky and Williams, 1993). Moreover, there is ample evidence that the flow inside hydrocyclones is anisotropic, causing the resistance to radial flow to become higher than that to axial flow (Duggins and Fritch, 1987).

The complexity of this flow has led designers to rely on the so-called design equations (Svarovsky, 1984). In designing a hydrocyclone for a particular application, these relationships are usually employed, which are based on correlations obtained experimentally. However, it is not always possible to count on such equations, since they may not be valid for the experimental conditions in question.
Alternatively, computational fluid dynamics techniques can be utilized to study and predict the dynamic behavior of hydrocyclones and cyclones. Besides providing a physical insight into the causes of the phenomena observed, this approach may allow the reliable design of these devices.

The main difficulty in simulating the dynamic behavior in a hydrocyclone without an air core is that the flow is known to be anisotropic, as mentioned before, and therefore the conventional k-e and Prandtl mixing length models cannot be applied. Indeed, several authors have shown the inaccuracy of these models in their standard form when employed to simulate the flow inside hydrocyclones (Malhotra et al, 1994) and air cyclones (Meier and Mori, 1999). Considerable effort has been expended in the adaptation of these models to account for anisotropic effects in cyclones and hydrocyclones and successful results have been reported (Malhotra et al, 1994; Meier and Mori, 1999 and Hsieh and Rajamani, 1991). However, the modifications in the turbulence models either increased the complexity of the models (Dyakowsky and Williams, 1993; Malhotra et al, 1994 and Meier and Mori, 1999) or required new constants to be fit or experimental information (Hsieh and Rajamani, 1991).

The present contribution aims to simulate the flow in a hydrocyclone using subgrid-scale modeling to account for the turbulence effects. This approach requires that the large, anisotropic eddies be resolved directly whereas the smallest, subgridscale eddies be modeled. As the small scales tend 
to be more nearly isotropic, this turbulence modeling needs not be revised like the classical models and appears to be suitable for simulating flows in hydrocyclones. The Smagorinsky model is the simplest subgrid-scale model and is employed in this work. A chief advantage of this model lies in its simplicity and dependence on a constant only, whose value ranges from 0.1 to 0.24 .

In order to compute the large scales of the flow, the full three-dimensional Navier-Stokes equations are solved with a finite-difference scheme which is second-order accurate in time. A fractional step method is employed to couple velocity and pressure and assure that the flow field is divergence-free at the end of each time step. The first-order upwind scheme is chosen for the convective terms because of its robustness. Grids as fine as 100000 nodes are employed to simulate Reynolds numbers ranging from 14300 to 26600. Numerical profiles and experimental data display fairly good agreement.

The numerical profiles obtained by the usage of the Smagorinsky model are compared with the experimental results obtained by Dabir (1983), who used Laser Doppler Anemometry. Dabir (1983) investigated the behavior of a water-fed Rietema hydrocyclone, whose dimensions are shown in Fig. 1 and Table 1, operating without an air core.

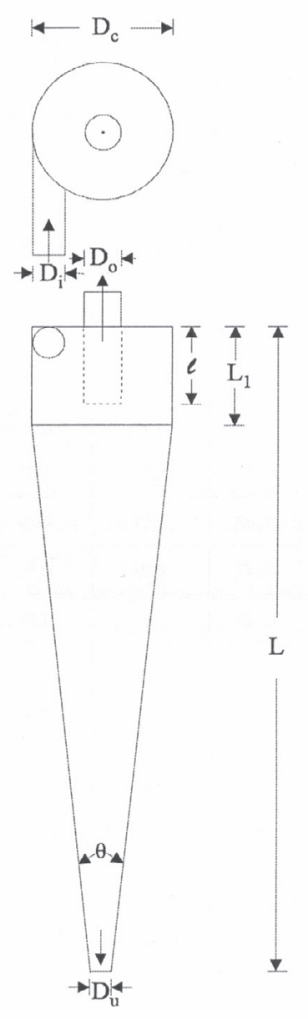

Figure 1. Schematics of a conventional hydrocyclone (Massarani, 1997).
Table 1. Dimensions of the hydrocyclone investigated.

\begin{tabular}{|c|c|}
\hline $\mathrm{D}_{\mathrm{c}}(\mathrm{m})$ & 0.0762 \\
\hline $\mathrm{D}_{\mathrm{o}}(\mathrm{m})$ & 0.0265 \\
\hline $\mathrm{D}_{\mathrm{i}}(\mathrm{m})$ & 0.0218 \\
\hline $\mathrm{D}_{\mathrm{u}}(\mathrm{m})$ & 0.0124 \\
\hline $\mathrm{L}(\mathrm{m})$ & 0.381 \\
\hline$\ell(\mathrm{m})$ & 0.0305 \\
\hline $\mathrm{L}_{1}(\mathrm{~m})$ & 0.0381 \\
\hline
\end{tabular}

\section{MATHEMATICAL MODEL}

Since Large Eddy Simulations are inherently three-dimensional and time-dependent, the mathematical model comprises the continuity equation and the full Navier-Stokes equations for incompressible flow in cylindrical coordinates. The singularity at the vertical axis $(\mathrm{r}=0)$ is treated as proposed by Verzicco and Orlandi al (1996). These equations must be filtered in order to separate the large scales and the subgrid scales (Lesieur, 1990).

This filtering process gives rise to additional stress tensors, which are modeled using the classical Boussinesq hypothesis. As a consequence, the viscosity in the filtered equations is the effective viscosity $\left(v_{\text {eff }}\right)$, given by the sum of the molecular viscosity $(\mathrm{v})$ and the turbulent viscosity $\left(v_{t}\right)$ :

$$
v_{\text {eff }}=v+v_{t}
$$

The turbulent viscosity in this work is computed with the Smagorinsky (1990) subgridscale model:

$$
\begin{aligned}
& v_{t}=\left(C_{s} d \Delta\right)^{2}\left(S_{i j} S_{i j}\right)^{1 / 2} \\
& \Delta=(r \Delta \theta \Delta r \Delta z)^{1 / 3}
\end{aligned}
$$

where $\mathrm{C}_{\mathrm{s}}$ is a constant between 0.1 and 0.24 related to the energy transfer from the large scales to the small ones. This constant depends on the flow type and must be tuned with experimental information. In this work, the experimental results have led the best value for $\mathrm{C}_{\mathrm{s}}$ to be 0.15 . $\mathrm{d}$ is the damping factor, $\Delta$ is the grid filter size, $\Delta \mathrm{r}, \Delta \mathrm{z}$ and $\Delta \theta$ are the grid spacings in the $r, z$ and $\theta$ directions, respectively and $S_{i j}$ is the strain rate, which is calculated with the resolved values of the filtered velocities. 
Because the turbulent viscosity must be zero on the wall, $\mathrm{C}_{\mathrm{s}}$ is multiplied by a damping factor which reads (Jacobsen, 1997):

$$
\begin{aligned}
& d=1-\exp \left(-r^{+} / 30\right)^{2} \\
& r^{+}=\frac{\left(r-r_{w}\right.}{v}\left(\frac{\tau_{w}}{\rho}\right)^{1 / 2}
\end{aligned}
$$

where $\tau_{\mathrm{w}}$ represents the shear stress on the wall, $\mathrm{r}-$ $r_{w}$ is the distance from the node to the nearest wall and $\rho$ is the fluid density.

No slip conditions have been used for the three velocity components on all the walls. In the experiments carried out by Dabir (1983), the flow ratio between the upper exit and the inlet was kept at $80 \%$. In order to keep the same flow ratio in the simulations, flat velocity profiles have been specified at the upper and lower exits. The velocities assigned were calculated from the respective flow rates at the outlets. At the inlet, the intersection between the inlet pipe and the cyclone was modeled as the flow inlet. The respective radial and tangential velocity components could then be determined based on the flow rate and the intersecting points.

The Reynolds number has been defined as:

$$
\operatorname{Re}=(4 Q) / \pi D_{i} v
$$

where $\mathrm{Q}$ is the inlet volumetric flow rate and $\mathrm{D}_{\mathrm{i}}$ is the inlet diameter of the cyclone.

\section{NUMERICAL METHOD}

The set of governing equations are solved by a fractional step method (Armfield and Street, 1999) on a nonuniform staggered grid. At each time step, the following steps are accomplished:

Predictor step:

$$
\frac{u_{i}{ }^{*}-u_{i}{ }^{n}}{\Delta t}=\frac{3}{2}\left[\begin{array}{l}
-\frac{\partial}{\partial x_{j}}\left(u_{j} u_{i}\right)+ \\
\frac{\partial}{\partial x_{j}}\left(v_{e f f} \frac{\partial u_{i}}{\partial x_{j}}+v_{\text {eff }} \frac{\partial u_{j}}{\partial x_{i}}\right)
\end{array}\right]^{n}+
$$

$$
-\frac{1}{2}\left[\begin{array}{l}
-\frac{\partial}{\partial x_{j}}\left(u_{j} u_{i}\right)+ \\
\frac{\partial}{\partial x_{j}}\left(v_{e f f} \frac{\partial u_{i}}{\partial x_{j}}+v_{\text {eff }} \frac{\partial u_{j}}{\partial x_{i}}\right)
\end{array}\right]^{n-1}
$$

Pressure calculation:

$$
\frac{\partial^{2} p^{n+1}}{\partial x_{i} \partial x_{i}}=\frac{1}{\Delta t} \frac{\partial u_{i}^{*}}{\partial x_{i}}
$$

Corrector step:

$$
\frac{u_{i}^{n+1}-u_{i}^{*}}{\Delta t}=-\frac{\partial p^{n+1}}{\partial x_{i}}
$$

The first-order upwind scheme and the central difference scheme are used to discretize the convective and the diffusive terms of the velocity components, respectively. The central difference scheme was experimented with for the convective terms, but it was not possible to bring the system to convergence. The equations were advanced explicitly in time with the $2^{\text {nd }}$ order AdamsBashforth scheme. The Poisson pressure equation has been solved by the strongly implicit procedure SIP (Stone, 1968) implemented by Norris (1996).

\section{RESULTS AND DISCUSSION}

\section{General flow patterns}

Figures 2(a-b) depict the instantaneous velocity vectors throughout the hydrocyclone $(\mathrm{Re}=26600)$ along the center axial-radial plane which bisects vertically across the inlet duct. The flow reversal is clearly seen along the entire conical section, as theoretically predicted (Svarovsky, 1984), as well as the presence of both outer and inner vortices. The flow is indeed asymmetric about the vertical axis in the vortex finder region, because the flow enters on one side of the vertical axis only. Below the vortex finder, the flow gradually becomes nearly symmetric, as the experiments by Hsieh and Rajamani (1991) revealed. Also, multiple reversals in flow direction exist in the region between the vortex finder and the wall. 

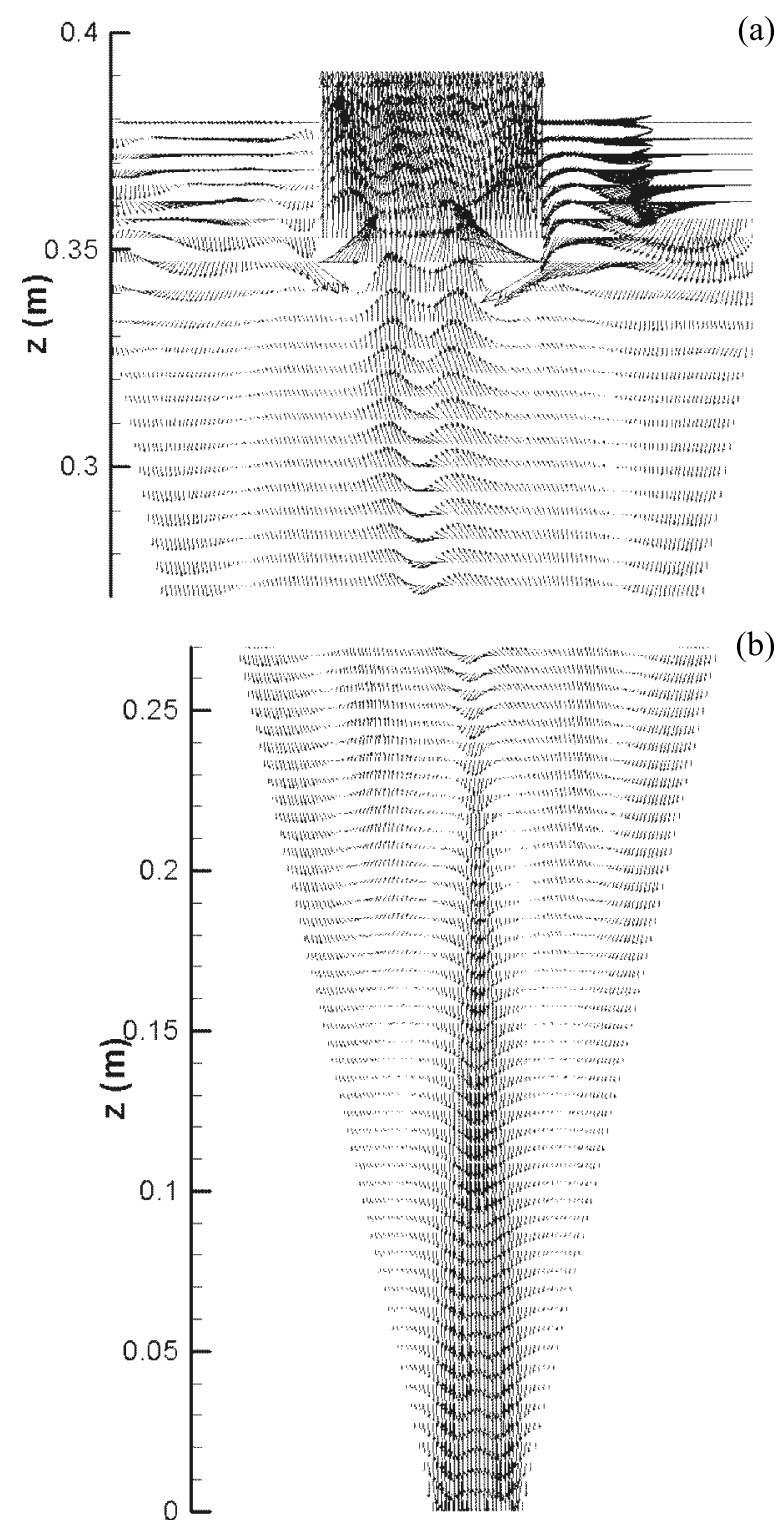

Figures 2(a-b). Velocity vectors along the center $\mathrm{r}-$ $\mathrm{z}$ plane $(\mathrm{Re}=26600)$.

This reverse flow continues throughout most of the hydrocyclone length. There is a secondary flow pattern at the top of the cylindrical section. It moves across the top cover to the base of the vortex finder and along its outer wall until it joins the rest of the fluid in the upper exit. This is referred to as short-circuit, which has a negative effect on the collection efficiency of coarse and fine particles.

Figure 3 presents the instantaneous velocity vectors in the upper section of the cyclone along a plane which is orthogonal to the plane shown in Fig. 2 for $R e=26600$. Recirculation eddies can be seen, some of which rotate in the opposite direction of those shown in Fig. 2. These recirculation eddies prevent any radial flow through a cylindrical surface within the flow so that it causes the resistance to radial flow to become higher than to axial flow. This effect tends to make the motion highly anisotropic. Figure 4 shows the instantaneous swirl velocity distribution for $\mathrm{Re}=26600$. It can be seen that it roughly distributes as coaxial cylinders. Such an effect is normally confirmed by independent experimental measurements (Hsieh and Rajamani, 1991) and is in accordance with Svarovsky (1994). It is known that in flows of this nature, the distribution of tangential velocity is a combination of a forced vortex (inner) and a free vortex (outer), resulting in the Rankine vortex (Jacobsen, 1997). The flow patterns for the other Reynolds numbers investigated were similar and hence will not be shown.

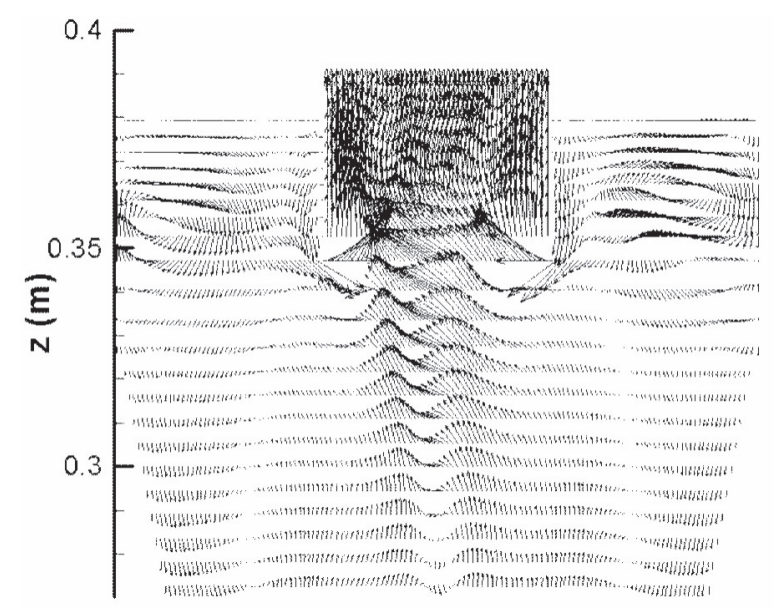

Figure 3. Velocity vectors along the $r-z$ plane orthogonal to plane shown in Fig. 2 ( $R e=26600)$.

\section{Comparison with experimental data}

Figure 5 compares the experimental data by Dabir (1983) for the swirl velocity at $\mathrm{Re}=24300$ and the results computed with the subgrid Smagorinsky model at $\mathrm{z}=0.32 \mathrm{~m}$, with the lines representing the computed results and the symbols the experimental data. The numerical profiles display a fairly good agreement with the experimental data, though underpredicting the swirl magnitude near the wall and slightly overpredicting it close to the peak. This is believed to be due to the low order scheme employed in this work as well as the relative coarseness of the grid. It is important to highlight that, unlike most RANS simulations, no wall functions have been used. 
Hence, it is expected that higher order schemes and finer grids will provide better agreement. Numerical experiments employing higher-order schemes are under way.

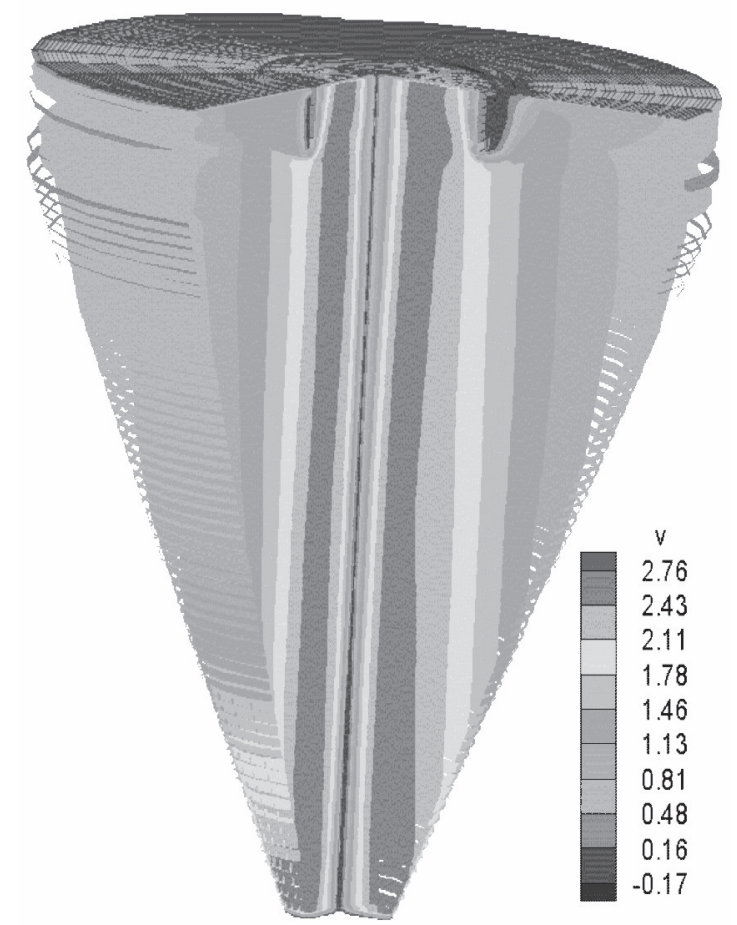

Figure 4. Swirl velocity contours (m/s) $R e=26600$

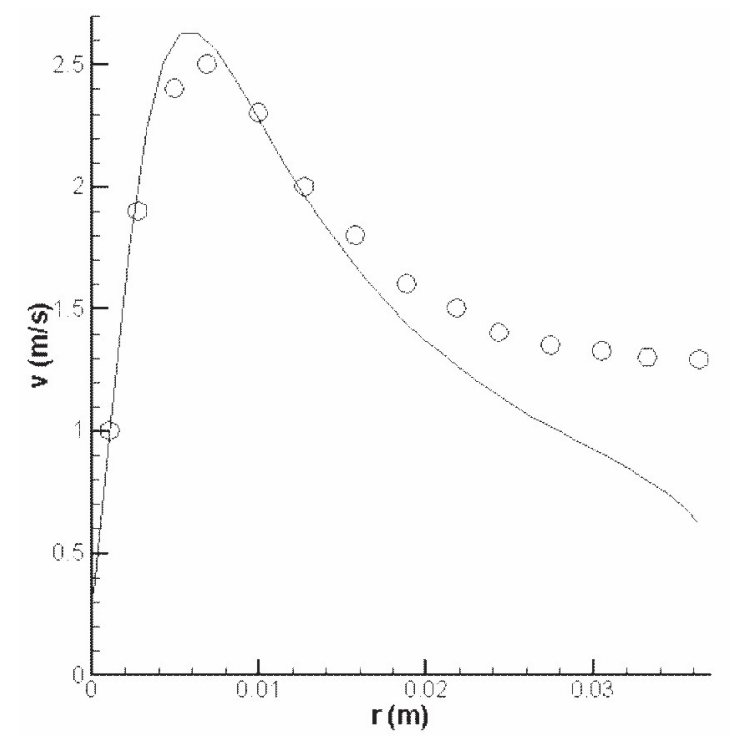

Figure 5. Comparison with experimental data by Dabir (1983) at $\mathrm{z}=0.32 \mathrm{~m}(\mathrm{Re}=24300)-$ line and circle represent the computed results and the experimental data, respectively.

Shown in Fig. 6 are the results of simulations for the time-averaged swirl velocities and experimental data at $\mathrm{z}=0.20 \mathrm{~m}$ at different Reynolds numbers. Once again, good agreement is found except near the wall. All the results presented in this work have utilized $\mathrm{Cs}=0.15$ associated to a grid resolution of nearly 100000 nodes. However, for each Reynolds number, it would be possible to fit values for Cs slightly different and refine the mesh in order to improve the agreement with experiments. This is a consequence of the fact that the Smagorinsky constant actually varies from place to place within the flow (Wilcox, 1994) and hence, the value tuned improves the overall agreement between simulated and experimental results at different turbulence levels.

A strict validation of the predicted results should also take into account axial velocity profiles. Even though such profiles are not available, accurate results for the tangential component suggest that the other components have been well predicted, as verified by Hsieh and Rajamani (1991). Since the experimental maximum pressure drops inside the hydrocyclone were available, these were compared to the values computed with the numerical model in Table 2. The agreement between simulated and experimental values of pressure drop is satisfactory, and it is hoped that the present methodology will be used in the near future as a tool for the study, design and optimization of hydrocyclones.

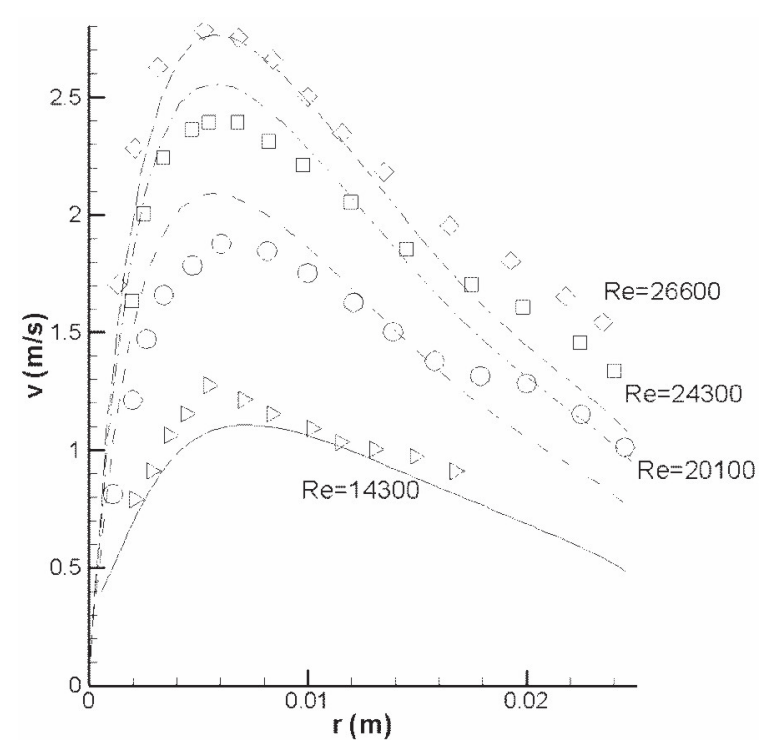

Figure 6. Comparison with experimental data by Dabir (1983) at $\mathrm{z}=0.20 \mathrm{~m}$ - lines and symbols represent the computed results and the experimental data, respectively.

Although the Smagorinsky model still presents the drawback of requiring the tuning of a 
constant, it provides advantages over turbulence models classically used for hydrocyclone simulation. In LES, the most anisotropic eddies are resolved directly, whereas only the smallest, less anisotropic eddies are modeled. Therefore, it is expected that the subgrid-scale models available, e.g. dynamic, mixed-scales, can be used in their standard form, unlike the k- $\varepsilon$ model, which requires further revision and possibly new constants.

Table 2. Experimental maximum pressure drop across the hydrocyclone (Dabir, 1983) compared to values computed with $\operatorname{LES}\left(\mathrm{C}_{\mathrm{s}}=0.15\right)$.

\begin{tabular}{|c|c|c|}
\hline $\operatorname{Re}$ & $\Delta \mathrm{p}_{\text {experimental }}\left(\mathrm{N} / \mathrm{m}^{2}\right)$ & $\Delta \mathrm{p}_{\text {computed }}\left(\mathrm{N} / \mathrm{m}^{2}\right)$ \\
\hline 14300 & 3330 & 3830 \\
\hline 20100 & 9000 & 9520 \\
\hline 24300 & 13000 & 14230 \\
\hline 26600 & 17800 & 16694 \\
\hline
\end{tabular}

\section{CONCLUSIONS}

The preliminary results of the application of subgrid-scale modeling to the flow in a hydrocyclone have shown that LES represents a promising alternative to models commonly employed to account for turbulence effects. The general trends of the variations have been consistently predicted with the Smagorinsky model in its standard form.

\section{ACKNOWLEDGEMENT}

The authors wish to thank $\mathrm{CNPq}$ for the financial support provided for this work.

\section{REFERENCES}

Armfield, S. and Street, R., 1999, The Fractional Step Method for the Navier-Stokes Equations on Staggered Grids: the Accuracy of Three Variations, Journal of Computational Physics, 153, pp. 660-665.

Dabir, B., 1983, Mean velocity measurements in a 3" hydrocyclone using Laser Doppler Anemometry, Ph.D. thesis, Chemical Engineering Department, Michigan State University, East Lansing, MI.
Duggins, R. K. and Fritch, P.C.W., 1987, Turbulence Effects in Hydrocyclones, Proceedings, in: $3 r d$ International Conference in Hydrocyclones, Oxford, pp. 75-81.

Dyakowsky, T. and Williams, R. A., 1993, Modelling Turbulent Flow within a small-diameter Hydrocyclone, Chemical Engineering Science, 48, n. 6 , pp. 1143-1152.

Hsieh, K. T. and Rajamani, R. K., 1991, Mathematical Model of the Hydrocyclone based on the Physics of Fluid Flow, AIChE Journal, 37, n. 5, pp. 735-745.

Jacobsen, C. B., 1997, Large Eddy Simulation of Confined Swirling Flow, Ph.D. thesis, Institute of Energy Technology, Aalborg University, Aalborg, Denmark.

Lesieur, M., 1990, Turbulence in Fluids, Kluwer Academic Publishers.

Malhotra, A., Branion, R. M. R. and Hauptmann, E. G., 1994, Modelling the Flow inside a Hydrocyclone, The Canadian Journal of Chemical Engineering, 72, pp. 953-960.

Massarani, G., 1997, Fluidodinâmica em Sistemas Particulados, Editora UFRJ, Rio de Janeiro.

Meier, H. F. and Mori, M., 1999, Anisotropic Behavior of the Reynolds Stress in Gas and Gas-Solid Flows in Cyclones, Powder Technology, 101, pp. 108-119.

Norris, S. E., 1996, An Investigation into the Comparative Speeds of Linear Solvers in the Solution of PDE's, Ph.D. thesis, School of Mechanical Engineering, University of South Wales, Australia.

Smagorinsky, J., 1963, General Circulation Experiments with the Primitive Equations, I. The basic experiment, Monthly Weather Review Rev 91, pp. 99-164.

Stone, H. L., 1968, Iterative Solution of Implicit Approximations of Multidimensional Partial Differential Equations, SIAM J. Numer. Anal., 5, n. 3, pp. 530-558.

Svarovsky, L., 1984, Hydrocyclones, Holt, Rinehart \& Winston Ltd, London.

Verzicco, R. and Orlandi, P., 1996, A FiniteDifference Scheme for Three-Dimensional Incompressible Flows in Cylindrical Coordinates, Journal of Computational Physics, 123, pp. 402414.

Wilcox, D. C., 1994, Turbulence modeling for $C F D$, DCW Industries, Inc. 